
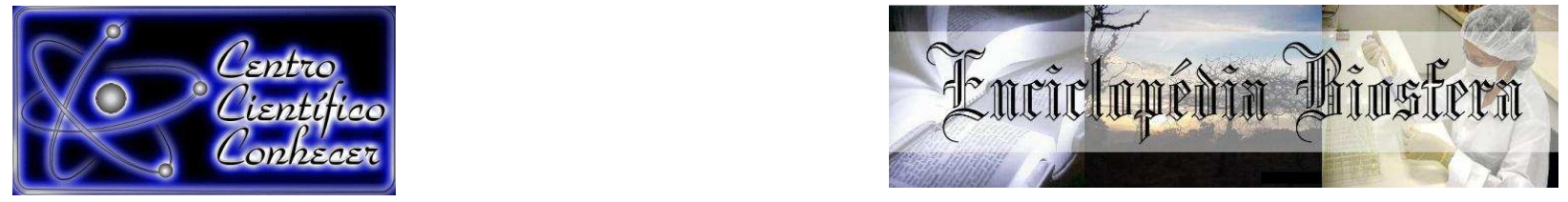

\title{
DIVERSIDADE DE GAFANHOTOS (ORTHOPTERA: ACRIDOMORPHA) DE UMA FLORESTA PRIMÁRIA DA AMAZÔNIA ORIENTAL
}

Carlos Elias de Souza Braga1', Ana Lúcia Nunes Gutjahr, José Wellington de Morais ${ }^{3}$

1 Professor Doutor, Centro de Ciências Sociais e Educação, Universidade do Estado do Pará (bragaelias@yahoo.com.br). Belém, Pará, Brasil.

2 Professora Doutora, Centro de Ciências Sociais e Educação, Universidade do Estado do Pará. Belém, Pará, Brasil.

3 Pesquisador Doutor, Coordenação de Biodiversidade do Instituto Nacional de Pesquisas da Amazônia. Manaus, Amazonas, Brasil.

Recebido em: 02/10/2017 - Aprovado em: 21/11/2017 - Publicado em: 05/12/2017 DOI: 10.18677/EnciBio_2017B81

\begin{abstract}
RESUMO
conhecimento sobre os insetos nas florestas tropicais ainda é incipiente ou inexistente para muitos grupos da entomofauna, principalmente os de grande diversidade. Este estudo se propõe a conhecer a composição faunística da comunidade de gafanhotos Acridoidea de uma floresta primária da Amazônia Oriental. Para isso, foram realizadas 36 expedições a Floresta Nacional de Caxiuanã, Portel, estado do Pará, $400 \mathrm{~km}$ a oeste de Belém (0157'36,82"S e $\left.51^{\circ} 36,47^{\prime} 61^{\prime \prime} \mathrm{W}\right)$, durante o período de março/2008 a fevereiro/2011. Os gafanhotos foram coletados com rede entomológica por quatro coletores experientes em duas trilhas, cada uma de $5 \mathrm{~km}$ por $1 \mathrm{~m}$, no sítio de amostragem do PPBio Amazônia Oriental. Foram coletados 10.541 gafanhotos pertencentes a três famílias, 8 subfamílias, 19 tribos, 40 gêneros e 51 espécies. Acrididae foi a família mais abundante $(71,7 \%)$ e Pyrgomorphidae a menos abundante $(3,5 \%)$. Entre as espécies amostradas, 10 correspondendo a $97,08 \%$ de todos os gafanhotos coletados. A riqueza de 51 espécies amostradas na Flona de Caxiuanã representou cerca de $5 \%$ dos gafanhotos amazônicos e 10\% da Amazônia Brasileira. As informações obtidas neste estudo correspondem a uma valorosa contribuição ao conhecimento da biodiversidade amazônica.
\end{abstract}

PALAVRAS-CHAVE: caxiuanã, Infraordem Acrididea, Inventário faunístico,

\section{DIVERSITY OF GRASSHOPPER (ORTHOPTERA: ACRIDOMORPHA) FROM A PRIMARY FOREST OF THE EASTERN AMAZON, PARÁ, BRAZIL}

\begin{abstract}
The knowledge about the insects from the tropical forest is still incipient or nonexistent for many grups on entomofauna, especially those of great diversity. This study intends to know the faunistic composition of the Acridoidea grasshopper community of a primary forest of the Eastern Amazon. For this, 36 expeditions were carried out to Flona de Caxiuanã, Portel, Pará state, $400 \mathrm{~km}$ west of Belém (015' 36.82 "S and 5136.47'61" W), during the period from March 2008 to February
\end{abstract}


/ 2011. Four experienced collectors collected the grasshoppers with a butterfly net on two trails, each $5 \mathrm{~km}$ by $1 \mathrm{~m}$, at the PPBio Amazona Oriental sampling area. 10,541 specimens of grasshopper were collected from three families, 8 subfamilies, 19 tribes, 40 genera and 51 species. The Acrididae family was the most abundant $(71.7 \%)$ and Pyrgomorphidae was the least abundant (3.5\%). Among the species sampled, 10 concentrated the most abundant, corresponding to $97.08 \%$ of all grasshoppers collected. The abundance of 51 species sampled in Flona de Caxiuanã, represented about $5 \%$ of locust in the Amazon region and $10 \%$ in the Brazilian Amazon. The information obtained in this study corresponds to a valuable contribution to the knowledge of the Amazonian biodiversity.

KEYWORDS: Infraorder Acrididea, Fauna inventory, Caxiuanã.

\section{INTRODUÇÃO}

$\mathrm{Na}$ Terra a maior diversidade de insetos se encontra nas florestas tropicais (THOMAZINI; THOMAZINI, 2000), onde poucos estudos têm sido realizados para caracterizar as comunidades desses invertebrados. Esse fato constitui um gargalo de conhecimento da biodiversidade tropical. Entretanto, outros fatores contribuem para as poucas informações da entomofauna dessas florestas, entre eles, destacamse: a falta de incentivo à pesquisa, a carência de taxonomistas para garantir a identificação das espécies inventariadas e a falta de padronização dos métodos de amostragem da entomofauna em geral. Este último fator é importante para possibilitar a comparação de informações em uma escala espaço-temporal, oriundas de diferentes regiões (LAMARRE et al., 2012). Todo esse cenário é fundamental para expor as dificuldades que impedem o conhecimento e o entendimento sobre a dinâmica que rege a biodiversidade existente nos diferentes ambientas tropicais.

Alguns grupos de insetos têm sido melhor estudados nas regiões tropicais, entretanto, ainda é necessário um esforço maior no que se refere aos grupos de insetos da Amazônia brasileira. Destaca-se, ainda, que apesar desses artrópodes desenvolverem papéis fundamentais para a manutenção e o bom funcionamento dos ecossistemas, eles são negligenciados nos grandes estudos regionais, sendo abordados apenas os grupos que possuem representantes vetores de doenças ou que sejam pragas agrícolas (PODEROSO et al., 2013), enquanto os insetos que atuam como polinizadores, predadores e saprófagos, por exemplo, são evitados.

Entre os insetos, os ortópteros são considerados o sexto maior grupo em quantidade de espécies no mundo, com mais de 27.000 espécies descritas (SONG et al., 2015; CIGLIANO et al., 2017), estes se caracterizam por apresentarem insetos com as pernas posteriores adaptadas para saltar. Os gafanhotos, grilos e as esperanças são os principais grupos dessa ordem (NUNES-GUTJAHR; BRAGA, 2010). Nesta ordem se destacam os gafanhotos verdadeiros pertencentes à superfamília Acridoidea e que se caracterizam por possuírem até 30 antenômeros (NUNES-GUTJAHR; BRAGA, 2016).

Atualmente, pouca informação se encontra disponível sobre os Orthoptera de florestas tropicais, embora existam alguns estudos sobre gafanhotos que vivem em associação com plantas aquáticas (BRAGA et al., 2011, FRANCESCHINI et al., 2013, CAPELLO; FRANCESCHINI, 2014, SILVA et al., 2014) e também que habitam o dossel das florestas. Além disso, Lamarre et al. (2012), admitem que as comunidades de gafanhotos de dossel parecem ter maior riqueza do que as do subbosque, o que possivelmente aumenta o nível de desconhecimento da entomofauna, devido à dificuldade para a execução de inventários neste estrato florestal. 
Portanto, conhecer a composição das espécies de um dado local é importante, visto que irá refletir a biodiversidade nos ecossistemas onde vive e principalmente para determinar os papeis ecológicos e sua importância no meio em que se encontra, sendo imprescindíveis, para isso, a execução de inventários de fauna nos ambientes de florestas. Desta forma, este estudo objetivou realizar um inventário faunístico de gafanhotos Acridomorfos, ocorrentes em uma floresta primária da Amazônia Oriental, com a finalidade de produzir dados sobre as comunidades desses insetos para ampliar o conhecimento da biodiversidade na Amazônia.

\section{Área de Estudo}

\section{MATERIAL E MÉTODOS}

O estudo foi desenvolvido no sítio de amostras do Programa de Pesquisa em Biodiversidade (PPBio), da Amazônia Oriental, na Floresta Nacional de Caxiuanã (015'36.82"S e 5136,47'61"W), que está distante cerca de $30 \mathrm{~km}$ de distância da Estação Científica Ferreira Penna e $400 \mathrm{~km}$ a oeste de Belém, Pará (SILVA et al., 2013). A Flona está localizada na Mesorregião do Marajó, nos municípios de Melgaço e Portel e possui 330.000 ha de área (LISBOA, 2013), sendo considerada a maior unidade de conservação no interflúvio dos rios Tocantins e Xingu (Figura 1).

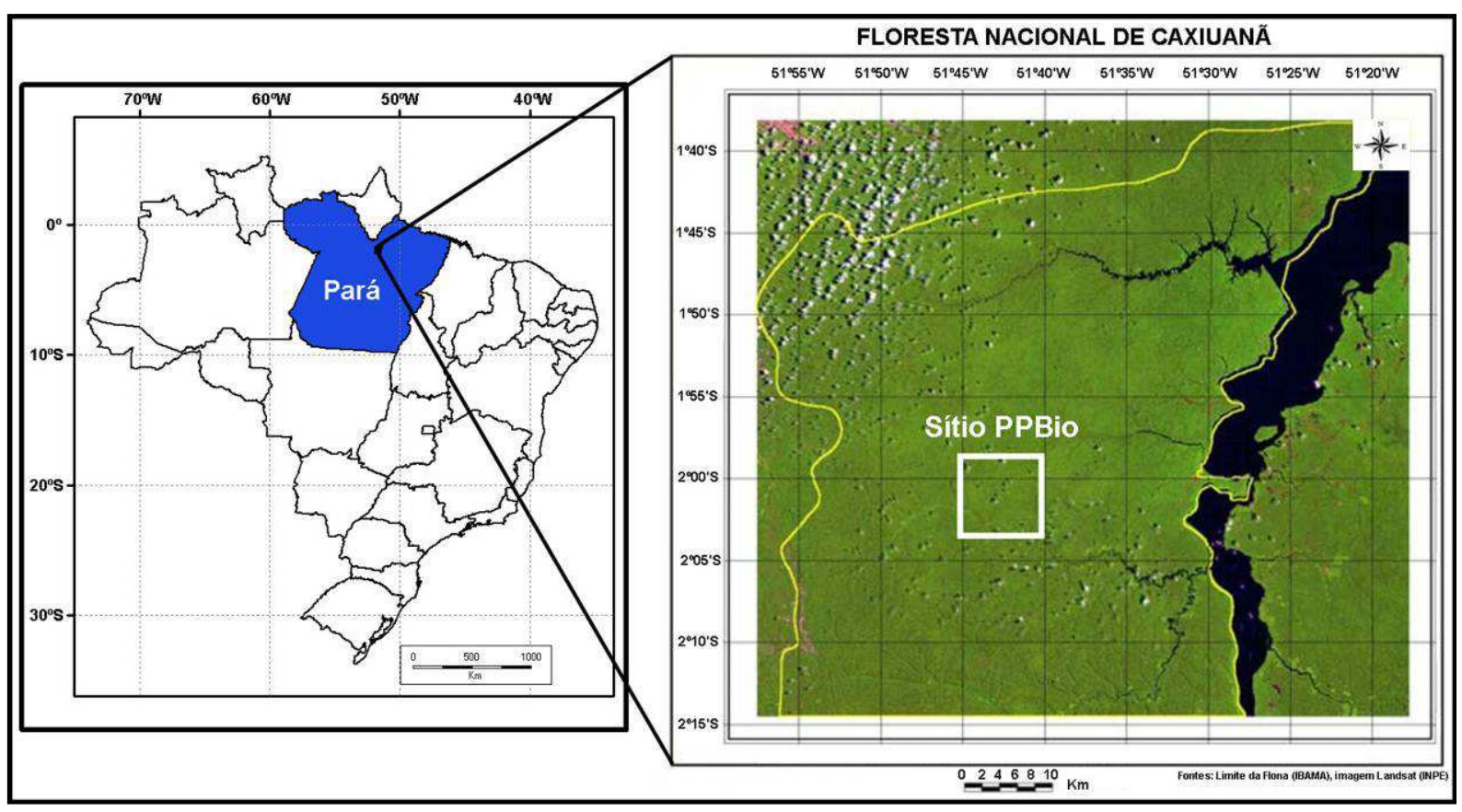

FIGURA 1. Localização do sítio do Programa de Pesquisas em Biodiversidade na Floresta Nacional de Caxiuanã, Pará, Brasil. Fonte: Os autores.

\section{Amostragem e método de coleta}

As coletas foram realizadas no período de março de 2008 a fevereiro de 2011, totalizando 36 excursões mensais. A área de amostragem correspondeu a duas trilhas da grade PPBio Amazônia-Oriental, designadas I (sentido norte/sul) e B (sentido leste/oeste) que apresentam (cada uma) $5.000 \mathrm{~m}$ de comprimento e $1 \mathrm{~m}$ de largura (PPBIO, 2012). As trilhas foram determinadas através de um sorteio (amostragem ao acaso simples), entre as 12 trilhas (A, B, C, D, E, F, G, H, I, J, L, M) existentes na Flona de Caxiuanã. 
A técnica de coleta utilizada foi a de captura ativa em campo, com o auxílio de rede entomológica, com aro de $40 \mathrm{~cm}$ de diâmetro, saco coletor de $60 \mathrm{~cm}$ de comprimento e cabo de madeira de $100 \mathrm{~cm}$ de comprimento. A amostragem de exemplares foi efetuada por quatro coletores treinados nessa técnica de captura. $O$ deslocamento nas trilhas I e B se deu a partir do ponto $0 \mathrm{~m}$ até $05.000 \mathrm{~m}$. O horário das coletas foi das $8 \mathrm{~h}$ às $16 \mathrm{~h}$, devido aos gafanhotos serem insetos de hábito diurno, sendo assim, pode-se capturar um número variado de indivíduos e espécies desses insetos (CARVALHO et al., 2014) desde o início ao final de suas atividades.

Os gafanhotos capturados foram depositados em sacos plásticos com etiquetas contendo as informações do ponto de captura e foram transportados para o laboratório, onde foram triados, montados e etiquetados. Os exemplares adultos foram montados em alfinetes entomológicos e as ninfas em meio líquido (álcool 80\%). Todo o material coletado foi identificado e incorporado aos acervos da Coleção Zoológica "Dr. Joachim Adis" da Universidade do Estado do Pará (UEPA) e da Coleção de Invertebrados do Museu Paraense Emílio Goeldi.

Para identificar os gafanhotos, utilizou-se estereomicroscópio (Leica MZ16) e chaves dicotômicas para espécies de gafanhotos. Além disso, foi feita a comparação com espécimes tipos da Coleção Entomológica Campos Seabra do Museu Nacional do Rio de Janeiro (MNRJ) e por fotos dos tipos disponíveis no portal Orthoptera Species File Online (http://Orthoptera.SpeciesFile.org), que é uma base mundial de dados taxonômicos das espécies válidas de Orthoptera, e que possui mais de 80.000 imagens (CIGLIANO et al., 2017).

\section{Análise de dados}

A análise dos dados foi realizada através da interpretação descritiva dos resultados e das curvas cumulativas das espécies em função dos estimadores não paramétricos Chao I e Chao II, Jackknife I e Jackknife II (GOTELLI; COLWELL, 2011), com intervalo de confiança de $95 \%$ e geradas pelo programa EstimateS 8.0 (COLWELL et al., 2012).

\section{RESULTADOS E DISCUSSÃO}

Foram coletados 10.541 gafanhotos pertencentes a três famílias, oito subfamílias, 19 tribos, 40 gêneros e 51 espécies (Tabela 1). Também, foi constatado que $39,1 \%(n=4.122)$ dos exemplares coletados encontravam-se no estágio adulto e $60,9 \%(n=6.419)$ no estágio de ninfa. Entre os gafanhotos adultos foi observado que a maioria da população $(56,1 \%)$ era de machos em comparação com as fêmeas $(43,9 \%)$. Para todo o período de coleta, a abundância média mensal de gafanhotos (adultos e imaturos) capturados foi de 293 indivíduos, com valor mínimo mensal de 162 exemplares e máximo de 473 exemplares, sendo que para os adultos foi de 115 indivíduos (31 - 244) e para as ninfas de 178 indivíduos (27 - 380).

Nas populações de muitos grupos de insetos, a maior ocorrência de imaturos, inclusive em ortópteros, já foi observada em outros estudos. Em relação a isso, evidencia-se que em uma comunidade de ortópteros (esperanças, gafanhotos e grilos) em uma floresta tropical da planície da ilha de Bórneu, na Malásia, a população de ninfas (imaturos) foi em torno de $90 \%$, porém, entre os gafanhotos esse índice foi de $80 \%$ de imaturos (FLOREN et al., 2001). Na Amazônia Central (BRAGA et al., 2011), verificaram a incidência de $65 \%$ imaturos, nas populações de gafanhotos semiaquáticos, reforçando que tal estratégia é comum nas comunidades de gafanhotos. 
A família mais numerosa foi Acrididae com 7.555 espécimes amostrados $(71,7 \%)$. A família Pyrgomorphidae teve a menor quantidade de indivíduos capturados ( $n=372 ; 3,5 \%$ ), enquanto que Romaleidae representou $24,8 \%$ ( $n=$ 2.614). A única família, com ocorrência registrada para o Brasil, não encontrada na Flona de Caxiuanã foi Ommexechidae que é representada por sete espécies amazônicas (CIGLIANO et al., 2017).

Quanto à riqueza, as 51 espécies amostradas na Flona de Caxiuanã, representam cerca de $5 \%$ dos gafanhotos amazônicos, $10 \%$ da Amazônia Brasileira (CIGLIANO et al., 2017), além de $43 \%$ das espécies do Estado do Pará (CARBONELL, 2010). Do total de espécies amostradas, 29 (56,9\%) pertenciam à Acrididae, $21(41,2 \%)$ a Romaleidae e uma espécie (1,9\%) a Pyrgomorphidae, sendo esses resultados equivalentes a $10 \%, 16 \%$ e $50 \%$, respectivamente, da Acridofauna amazônica para cada uma das famílias inventariadas (CARBONELL, 2010).

A grande ocorrência de Acrididae é explicada por se tratar da família que possui a maior riqueza entre os ortópteros, com 6.576 espécies, e a que possui os gafanhotos encontrados com maior frequência nos ambientes naturais, tais como observado em estudos realizados no Cerrado (GUERRA et al., 2012), na Amazônia (NUNES-GUTJAHR; BRAGA, 2010, 2015) e em áreas de fragmentos florestais e de cultivares no Sul do Brasil (LUTINSKI et al., 2011).

As espécies mais numerosas foram Clematodina eckardtiana Günther, 1940 $(\mathrm{n}=6.085)$, Colpolopha obsoleta (Serville, 1831) $(\mathrm{n}=2.087)$, Omura congrua Walker, $1870(n=372)$, Copiocera surinamensis Rehn, $1913(n=359)$, Phaeoparia lineaalba lineaalba (Linnaeus, 1758) $(\mathrm{n}=290)$, Tetrataenia surinama (Linnaeus, 1764) $(n=262)$, Locheuma brunneri (Scudder, 1875) $(n=251)$, Coscineuta marginalis (Walker, 1870) $(\mathrm{n}=194)$, Syntomacris virgata (Gerstaecker, 1889) $(\mathrm{n}=$ 187) e Epiprora hilaris (Gerstaecker, 1889) $(n=146)$ que juntas corresponderam a $97,08 \%$ dos espécimes amostrados (Tabela 1 ).

Em estudos sobre a Acridofauna é evidenciado que as espécies dominantes, apesar de representarem uma pequena parcela da riqueza de gafanhotos tendem a apresentar grandes valores de abundância. No Pampa Argentino, Mariottini et al. (2011), constataram que apenas seis espécies representavam juntas $91,1 \%$ dos indivíduos da comunidade de gafanhotos daquele bioma, na Mata Atlântica Lutinski et al. (2009) verificaram que 10 espécies equivaliam a $80,6 \%$ dos exemplares, enquanto que na Amazônia, Nunes-Gutjahr e Braga (2010), observaram que 11 espécies correspondiam a $89,4 \%$ dos gafanhotos inventariados.

TABELA 1. Lista taxonômica (Famílias, subfamílias, tribos, gêneros, espécies) e total de exemplares coletados na Flona de Caxiuanã no período de março de 2008 a fevereiro de 2011.

\begin{tabular}{|c|c|c|c|}
\hline $\begin{array}{l}\text { Família / } \\
\text { Subfamília }\end{array}$ & Tribo & Gênero / Espécie & $\begin{array}{l}\text { Número de } \\
\text { exemplares }\end{array}$ \\
\hline \multicolumn{4}{|l|}{ ACRIDIDAE } \\
\hline \multirow[t]{3}{*}{ Copiocerinae } & Copiocerini & Copiocera prasina & 67 \\
\hline & & Copiocera surinamensis & 359 \\
\hline & & Copiocerina formosa & 17 \\
\hline \multirow[t]{3}{*}{ Leptysminae } & Chloropseustini & Chloropseustes leucotylus & 41 \\
\hline & Tetrataeniini & Cornops aquaticum & 1 \\
\hline & & Tetrataenia surinama & 262 \\
\hline Melanoplinae & Dichroplini & Baeacris punctulata & 1 \\
\hline
\end{tabular}

ENCICLOPÉDIA BIOSFERA, Centro Científico Conhecer - Goiânia, v.14 n.26; p.952 


\begin{tabular}{|c|c|c|c|}
\hline \multirow[t]{10}{*}{ Ommatolampidinae } & \multirow[t]{2}{*}{ Abracrini } & Abracris flavolineata & $\begin{array}{l}2 \\
3\end{array}$ \\
\hline & & $\begin{array}{l}\text { Psiloscirtus olivaceus } \\
\text { Eusitalces vittatus }\end{array}$ & $\begin{array}{l}3 \\
2\end{array}$ \\
\hline & \multirow[t]{2}{*}{ Clematodinini } & Clematodina eckardtiana & 6.085 \\
\hline & & Rehnuciera fuscomaculata & 27 \\
\hline & \multirow[t]{2}{*}{ Ommatolampidini } & Anablysis guyoti & 2 \\
\hline & & Locheuma brunneri & 251 \\
\hline & \multirow[t]{4}{*}{ Syntomacrini } & Caloscirtus cardinalis & 14 \\
\hline & & Oyampiacris nemorensis & 4 \\
\hline & & Pseudanniceris nigrinervis & 1 \\
\hline & & Syntomacris virgata & 187 \\
\hline \multirow[t]{10}{*}{ Proctolabinae } & Coscineutini & Coscineuta marginalis & 195 \\
\hline & \multirow[t]{9}{*}{ Proctolabini } & Eucephalacris brasiliensis & 4 \\
\hline & & Eucephalacris paraensis & 1 \\
\hline & & Eucephalacris spatulicerca & 1 \\
\hline & & Eucerotettix ludificator & 1 \\
\hline & & Dendrophilacris boulardi & 6 \\
\hline & & Halticacris orientalis & 10 \\
\hline & & Poecilocloeus modestus & 3 \\
\hline & & Poecilocloeus collaris & 5 \\
\hline & & Saltonacris phantastica & 3 \\
\hline \multicolumn{4}{|c|}{ PYRGOMORPHIDAE } \\
\hline Pyrgomorphinae & Omurini & Omura congrua & 372 \\
\hline \multirow{10}{*}{ Bactrophorinae } & & & \\
\hline & \multirow{8}{*}{ Ophthalmolampini } & $\begin{array}{l}\text { Adrolampis arrogans } \\
\text { Apophylacris incodita }\end{array}$ & $\begin{array}{l}1 \\
5\end{array}$ \\
\hline & & Ophthalmolampis fervida & 24 \\
\hline & & Ophthalmolampis occulata & 19 \\
\hline & & Ophthalmolampis truculenta & 6 \\
\hline & & Pseudonautia biguttata & 2 \\
\hline & & Pseudonautia remota & 3 \\
\hline & & Pseudonautia saltuensis & 1 \\
\hline & & Pseudonautia tinctifemur & 2 \\
\hline & Taeniophorini & Taeniophora sp. & 6 \\
\hline \multirow[t]{11}{*}{ Romaleinae } & Hisychiini & Pseudhisychius brasiliensis & 8 \\
\hline & \multirow[t]{2}{*}{ Phaeopariini } & Epiprora hilaris & 146 \\
\hline & & $\begin{array}{l}\text { Phaeoparia lineaalba } \\
\text { lineaalba }\end{array}$ & 290 \\
\hline & Procolpini & Aeolacris caternaultii & 1 \\
\hline & \multirow[t]{4}{*}{ Romaleini } & Aprionacris fissicauda & 2 \\
\hline & & Chariacris dulcis & 6 \\
\hline & & Chromacris speciosa & 1 \\
\hline & & Colpolopha obsoleta & 2087 \\
\hline & \multirow[t]{2}{*}{ Tropidacrini } & Titanacris albipes & 1 \\
\hline & & Titanacris picticrus picticrus & 2 \\
\hline & Trybliophorini & $\begin{array}{l}\text { Trybliophorus } \\
\text { octomaculatus }\end{array}$ & 1 \\
\hline
\end{tabular}

\begin{tabular}{lllll}
\hline TOTAL & $3 / 8$ & 19 & $40 / 51$ & 10.541
\end{tabular}


Para analisar o esforço de amostragem empregado (WANDSCHEER et al., 2016), foi realizada a análise da curva acumulativa de espécies para os estimadores Chao I $(n=60)$ e Chao II $(n=60)$ e Jackknife I $(n=64)$ que indicaram os valores mais próximos ao observado $(n=51)$, enquanto que Jackknife II $(n=70)$ estimou a riqueza mais elevada. Entretanto, nota-se que as curvas de acumulação apresentam uma tendência de estabilização, apesar de não ter atingido a assíntota, o que leva a considerar que o esforço de amostragem utilizado neste estudo foi satisfatório, principalmente, porque a riqueza observada (Sobs $=51$ espécies) para comunidade de gafanhotos representou $85 \%$ do esperado para os estimadores Chao I e II, $80 \%$ para Jackknife I e 73\% para Jackknife II (Figura 2). Segundo Amédégnato (2003) o método de busca ativa em transectos é considerado o mais eficiente para coleta de gafanhotos no sub-bosque das florestas tropicais.

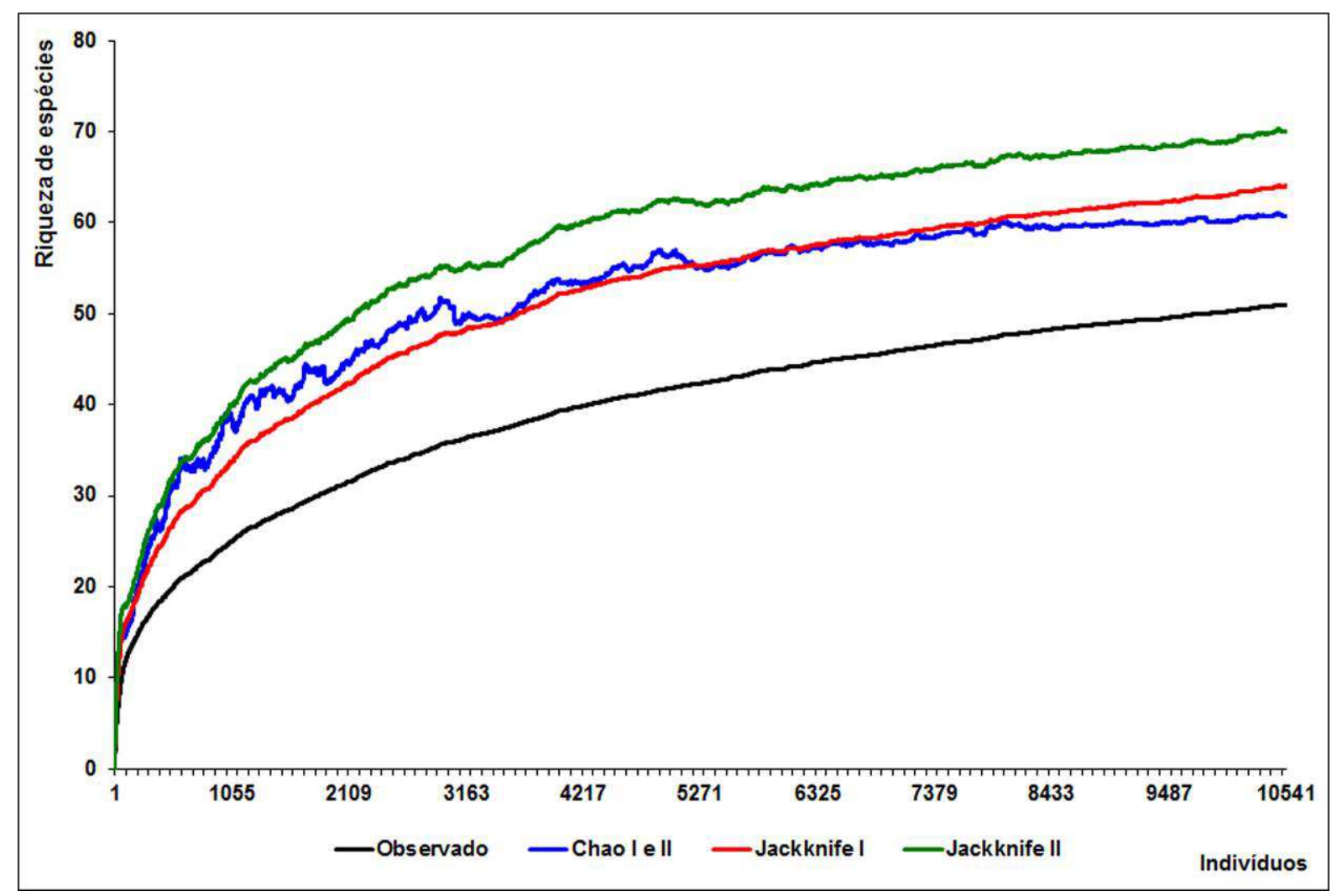

FIGURA 2. Curva acumulativa em função do indivíduo, para as espécies de gafanhotos, coletadas na Floresta Nacional de Caxiuanã no período de março de 2008 a fevereiro de 2011. Fonte: Os autores.

Ao se analisar o período de coleta em separado ( $1^{\circ}$ ano, $2^{\circ}$ ano e $3^{\circ}$ ano), percebeu-se que os estimadores de riqueza tendem a ter uma maior proximidade com os valores observados ao final do estudo (Tabela 2). Entre as 51 espécies amostradas, 22 foram coletadas em todos os anos. No primeiro ano foram coletados 3.230 exemplares de 39 espécies, sendo sete exclusivas para o período, no segundo ano foram 3.266 exemplares de 35 espécies e quatro exclusivas, no terceiro ano foram 4.057 exemplares de 34 espécies e cinco exclusivas.

A taxa de incremento a cada ano de amostragem foi em média de seis espécies e a riqueza média acumulada de 45 espécies, dessa forma, o valor máximo estimado de 70 espécies (estimador Jackknife II) poderia ser atingido em 
aproximadamente três anos. Entretanto, sabe-se que a curva de acumulação de espécies é diretamente influenciada pelas características dos ambientes amostrados (THOMPSON et al., 2003), bem como pelas espécies pouco abundantes nas amostras (KAMINSKI et al., 2016) e que com o aumento do esforço de amostragem, há a tendência, ainda que mínima, de os valores estimados crescerem, principalmente em florestas tropicais, devido a grande riqueza de espécies nesses ecossistemas (SCHILLING; BATISTA, 2008).

TABELA 2 Valores de riqueza observada, estimada e acumulada e taxa de incremento das espécies da comunidade de gafanhotos na Flona de Caxiuanã no período de março de 2008 a fevereiro de 2011.

\begin{tabular}{|c|c|c|c|c|c|}
\hline \multirow{2}{*}{ Amostragem } & \multirow{2}{*}{ Observado } & \multicolumn{4}{|c|}{ Estimadores } \\
\hline & & Chao I & Chao II & Jackknife I & Jackknife II \\
\hline $1^{\circ} \mathrm{ANO}$ & 39 & 47 & 47 & 51 & 56 \\
\hline $2^{\circ}$ ANO & 35 & 48 & 48 & 47 & 55 \\
\hline $3^{\circ}$ ANO & 34 & 51 & 51 & 46 & 55 \\
\hline Riqueza total & 51 & 60 & 60 & 64 & 70 \\
\hline Período & \multicolumn{2}{|c|}{ Riqueza acumulada } & \multicolumn{3}{|c|}{ Taxa de incremento de espécies } \\
\hline $1 \stackrel{\circ}{ }$ ANO & \multicolumn{2}{|c|}{39} & \multicolumn{3}{|c|}{-} \\
\hline $1^{\circ}+2^{\circ}$ ANO & \multicolumn{2}{|c|}{46} & \multicolumn{3}{|c|}{7} \\
\hline $1^{\circ}+2^{\circ}+3^{\circ}$ ANO & \multicolumn{2}{|c|}{51} & \multicolumn{3}{|c|}{5} \\
\hline Média & \multicolumn{2}{|c|}{45} & \multicolumn{3}{|c|}{6} \\
\hline
\end{tabular}

\section{CONCLUSÃO}

Acrididae correspondeu à maioria riqueza e abundância dos gafanhotos encontrados na Floresta Nacional de Caxiuanã. As espécies mais abundantes corresponderam a quase totalidade dos espécimes amostrados durante o período em estudo. A Flona possui uma grande representatividade da riqueza de gafanhotos com registro de ocorrência para o Pará e na Amazônia Brasileira, o que reforça a importância da manutenção nessa Unidade de Conservação brasileira. O esforço de amostragem utilizado para a realização do estudo foi satisfatório haja vista que os valores dos estimadores de riqueza foram muito próximos ao observado.

\section{AGRADECIMENTOS}

Os autores agradecem ao Programa de Pesquisas em Biodiversidade (PPBio), Museu Paraense Emílio Goeldi e ao projeto Cenários pelo apoio financeiro e logistico e, ao Conselho Nacional de Desenvolvimento Científico e Tecnológico (CNPq) pelo apoio financeiro referente ao Projeto Universal CNPq/2007 (Processo n-482955/2007-4): Sazonalidade e diversidade da Acridofauna (OrthopteraAcridoidea) em um sítio do Programa de Pesquisa em Biodiversidade da Amazônia Oriental - PPBio-Caxiuanã, Melgaço, Pará, o qual possibilitou a realização do inventário biológico deste estudo. 


\section{REFERÊNCIAS}

AMÉDÉGNATO, C. Microhabitat distribution of forest grasshoppers in the Amazon. In: BASSET, Y.; NOVOTNY, V.; MILLER, S. E.; KITCHING, R. L. Arthropods of tropical forests: spatio-temporal dynamics and resource use in the canopy. Cambridge University Press, Cambridge, 2003, p. 237-255.

BRAGA, C. E.; NUNES, A. L.; MORAIS, J. W.; ADIS, J. Fenologia de Cornops aquaticum (Bruner 1906) (Orthoptera: Acrididae) associado à Eichhornia crassipes (Mart.) Solms (Pontederiaceae) em um lago de Várzea na Amazônia Central, Brasil. Revista de la Sociedad Entomológica Argentina, v. 70, p. 185-196, 2011.

CAPELLO, S.; FRANCESCHINI, M. C. Number and duration of the juvenil e instars of the neotropical grasshopper Cornops aquaticum (Acrididae: Leptysminae) in Argentina. Interciencia, v. 39, n. 4, p. 255-259, 2014. Disponível em:< http://www.redalyc.org/pdf/339/33930412'007.pdf >

CARBONELL, C. S. Checklist of The Neotropical Acridomorph Species. Facultad de Ciencias, Universidad de la República, Montevideo, UY. Unpublished, 2010.

CARVALHO, N. L.; COSTA, E. C.; GARLET, J.; SOUZA, D. B.; BOSCARDIN, J. Horário Ideal para Coleta de Gafanhotos na Depressão Central no Rio Grande do Sul. EntomoBrasilis, v. 7, n. 2, p. 93-98, 2014.

CIGLIANO, M. M., H. BRAUN, D. C. EADES \& D. OTTE. Orthoptera Species File. Version 5.0/5.0. Disponível em: <http://Orthoptera.SpeciesFile.org>. Acesso em: 16 de set. 2017.

COLWELL, R. K.; CHAO, A.; GOTELLI, N. J.; LIN, S. Y.; MAO, C. X.; CHAZDON. R. L.; LONGINO, J. T. Models and estimators linking individual-based and samplebased rarefaction, extrapolation, and comparison of assemblages. Journal of Plant Ecology, v. 5, p. 3-21, 2012.

FLOREN, A.; RIEDE, K.; INGRISCH, S. Diversity of Orthoptera from Bornean lowland rainforest trees. Ecotropica, v. 7, p. 33-42, 2001.

FRANCESCHINI, M. C.; WYSIECKI, M.L.; POI, A. Age Structure and Feeding of the Neotropical Grasshopper Cornops aquaticum (Bruner) (Orthoptera: Acrididae) on Water Hyacinth. Neotropical Entomology, v. 42, n. 4, p. 344-350, 2013. Disponível em:<https://doi.org/10.1007/s13744-013-0130-8> doi: 10.1007/s13744-013-0130-8

GOTELLI, N. J, COLWELL, R. K. Estimating species richness. In: Magurran, A.E.; McGill, B.J. Frontiers in Measuring Biodiversity. New York: Oxford University Press, p. 39-54, 2011.

GUERRA, W. D.; OLIVEIRA, P. C.; PUJOL-LUZ, J. R. Gafanhotos (Orthoptera Acridoidea) em áreas de cerrados e lavouras na Chapada dos Parecis, Estado de Mato Grosso, Brasil. Revista Brasileira de Entomologia, v. 56, p. 228-239, 2012.

KAMINSKI, N.; ANGELO, A. C.; NICOLA, P. A. A influência do gradiente sucessional e da frutificação de Merostachys aff. multiramea em uma comunidade de aves da 
Floresta com Araucária. Iheringia, Série Zoológica [online], v. 106, p. 1-9, 2016. Disponível em: <http://dx.doi.org/10.1590/1678-4766e2016002> doi: 10.1590/1678$4766 \mathrm{e} 2016002$

LAMARRE, G. P. A.; MOLTO, Q.; FINE, P. V. A.; BARALOTO, C. A. Comparison of two common flight interception traps to survey tropical arthropods. Zookeys, v. 216, p.43-55, 2012.

LISBOA, P.L.B. Caxiuanã: paraíso ainda preservado. MPEG, Belém, PA, BR. 2013.

LUTINSKI, C. J.; GARCIA, F. R. M.; COSTA, M. K. M.; LUTINSKI, J. A. Flutuação populacional de gafanhotos na Floresta Nacional de Chapecó, Santa Catarina. Ciência Rural, v.39, n.2, p. 555-558, 2009.

LUTINSKI, C.J.; LUTINSKI, J.A.; COSTA, M.K.M.; GARCIA, F.R.M. Análise faunística de gafanhotos na Floresta Nacional de Chapecó, Santa Catarina. Pesquisa Florestal Brasileira, v. 31, p. 43-50, 2011.

MARIOTTINI, Y.; WYSIECKI, M. L.; LANGE, C. E. Seasonal Occurrence of Life Stages of Grasshoppers (Orthoptera: Acridoidea) in the Southern Pampas, Argentina. Zoological Studies, v. 50, n. 6, p. 737-744, 2011.

NUNES-GUTJAHR, A. L.; BRAGA, C. E. Similaridade entre amostras da acridofauna (Orthoptera: Acrididae) em quatro áreas ao longo da estrada Santarém-Cuiabá (BR163), Pará, Brasil. Revista Nordestina de Zoologia, v. 4, n. 1, p. 118-130, 2010.

NUNES-GUTJAHR, A. L.; BRAGA, C. E. Análise faunística de gafanhotos Acridoidea da Volta Grande do Rio Xingu, área de influência direta da Hidrelétrica Belo Monte, Pará, Brasil. Revista Ciência Rural, v. 45, n. 7, p. 1220-1227, 2015.

NUNES-GUTJAHR, A. L.; BRAGA, C. E. Os gafanhotos Acridoidea (Orthoptera: Caelifera) da Coleção Zoológica Didático-Científica Dr. Joachim Adis da Universidade do Estado do Pará. Enciclopédia Biosfera, v. 13, n. 24, p.1250-1262, 2016.

PODEROSO, J. C. M.; COSTA, M. K. M.; CORREIA-OLIVEIRA, M. E.; DANTAS, P. C; ZANUNCIO, J. C.; RIBEIRO, G. T. Occurrence of Tropidacris collaris (Orthoptera: Acridoidea: Romaleidae) Damaging Casuarina glauca (Casuarinaceae) Plants in the Municipality of Central Bahia, Brazil. Florida Entomologist, v. 96, p. 268-269, 2013.

PROGRAMA DE PESQUISA EM BIODIVERSIDADE (PPBIO). 2012. Metadados Instalação da Grade ou Módulo RAPELD. Disponível em: <http://ppbio.inpa.gov.br/instalacao/grade>. Acesso em: 16 de set. 2017.

SCHILLING, A. C.; BATISTA, J. L. F. Curva de acumulação de espécies e suficiência amostral em florestas tropicais. Revista Brasileira de Botânica, v. 31, n. 1, p. 179$187,2008$. 
SILVA, M. M. F.; BASTOS, M. N. C.; GURGEL, E. S. C. Macrolobium Schreb. (Leguminosae, Caesalpinioideae) na Floresta Nacional de Caxiuanã, Pará, Brasil. Boletim do Museu Paraense Emílio Goeldi, v.8, n.1, p.75-93, 2013.

SILVA, F. R. J.; BATTIROLA, L. D.; LHANO, M. G.; SOUSA, W. O.; MARQUES, M.I. Morphometry of Cornops aquaticum (Orthoptera: Acrididae: Leptysminae) in the Pantanal of Mato Grosso, Brazil. Brazilian Journal of Biology, v. 74, n. 3, p. 730738, 2014. Disponível em: <http://dx.doi.org/10.1590/bjb.2014.0068> doi: $10.1590 / b j b .2014 .0068$

SONG, H.; AMÉDÉGNATO, C.; CIGLIANO, M. M.; DESUTTER-GRANDCOLAS, L.; HEADS, S. W.; HUANG, Y.; OTTE, D.; WHITING, M. F. 300 million years of diversification: elucidating the patterns of orthopteran evolution based on comprehensive taxon and gene sampling. Cladistics, p. 1-31, 2015.

THOMAZINI, M. J.; THOMAZINI, A. P. B. W. A fragmentação florestal e a diversidade de insetos nas florestas tropicais úmidas. Embrapa Acre, 21p, 2000.

THOMPSON, G. G.; WITHERS, P. C.; PIANKA, E. R.; THOMPSON, S. A. Assessing biodiversity with species accumulation curves; inventories of small reptiles by pittrapping in Western Australia. Austral Ecology, v. 28, p. 361-383, 2003.

WANDSCHEER, A. C. D.; BAUMART, J. S.; MARCHESAN, E.; SANTOS, S.; AVILA, L. A.; SOARES, C. F.; PIRES, M. M. Suficiência amostral para estudos de impacto ambiental sobre a comunidade de macroinvertebrados bentônicos em arrozais irrigados. Ciência Rural, v. 46, n. 1, p. 26-29, 2016. Disponível em:< http://dx.doi.org/10.1590/0103-8478cr20131218> doi: 10.1590/01038478 cr20131218. 